\title{
Effect of symmetry center losses on energy bands and carrier kinematics in $\mathrm{Zn}_{3} \mathrm{As}_{2}$ and $\mathrm{Cd}_{3} \mathrm{As}_{2}$
}

\author{
G. Chuiko, N. Don, V. Martyniuk, and D. Stepanchikov \\ Kherson National Technical University, Department of General and Applied Physics \\ 24,Berislavskoye shosse, 73008 Kherson,Ukraine, e-mail: gp47@mail.ru
}

\begin{abstract}
The comparative analysis of the band structure and carrier kinematics for $n$ $\mathrm{Cd}_{3} \mathrm{As}_{2}$ and $p-\mathrm{Zn}_{3} \mathrm{As}_{2}$ has been executed. The influence of presence and absence of symmetry center in different crystalline phases of the above materials is explored. The direct and indirect solutions of dispersion equations were used for the analysis. The results of researches are presented in the form of graphic dependences. The splitting of energy bands caused by the symmetry center loss is estimated. Such splitting is maximal along any directions normal to the main crystalline axis. The principle possibility of the carrier separation by spin with the use of found distinction for the modules of their velocities is shown.
\end{abstract}

Keywords: dispersion law, band structure, cadmium arsenide, zinc arsenide.

Manuscript received 24.01.06; accepted for publication 29.03.06.

\section{Introduction}

$\mathrm{Cd}_{3} \mathrm{As}_{2}$ and $\mathrm{Zn}_{3} \mathrm{As}_{2}$ have the equivalent polymorphic transformations below $500 \mathrm{~K}$ with the loss of centers of symmetry. As a result, the symmetry is lowered from $4 / \mathrm{mmm}\left(D_{4 h}\right)$ to $4 \mathrm{~mm}\left(C_{4 v}\right)$. Both crystals possess very similar lattices with 160 atoms per a cell, demonstrating the small tetragonal tensions: $0<\eta-1=\frac{c}{2 a}-1<<1$ [1]. Besides, their cells can be considered only as slightly divergent, if comparing the pair of crystal modifications with $4 / \mathrm{mmm}\left(D_{4 h}\right)$ and $4 m m\left(C_{4 v}\right)$ symmetries, not only without but with the center of symmetry, making it individual for each of both materials, of course. These cells differ only by one of the four layered atom packets, which create such a cell [2].

However, these materials possess also a few essential distinctions, despite the undoubted similarity of their crystalline forms. Firstly, there is a difference between the standard and inverted band structures: $\varepsilon_{g}>0$ for $\mathrm{Zn}_{3} \mathrm{As}_{2}$ and $\varepsilon_{g}<0$ for $\mathrm{Cd}_{3} \mathrm{As}_{2}$. Secondly, there is a contrast of their types of the conductivity: $p$ type and $n$-type, respectively $[1,2]$. As a result, the typical concentrations and carrier mobility differ within a few orders: these are much higher for $\mathrm{Cd}_{3} \mathrm{As}_{2}$.
Therefore, $\mathrm{Zn}_{3} \mathrm{As}_{2}$ is a typical semiconductor, whereas $\mathrm{Cd}_{3} \mathrm{As}_{2}$ looks like a semimetal. Thus, the proper Fermi levels are located not only within visibly different energy intervals but even within different energy bands of both materials.

Recently the simple band model was proposed for $\mathrm{Cd}_{3} \mathrm{As}_{2}$ [3]. On the one hand, this model is a generalization of well-known Kane's and Kildal's models $[4,5]$. On the other hand, the model [3] enables to take into account the symmetry center loss and the small tetragonal tensions. Moreover, this theory can be appropriately used both for semiconductors $\left(\varepsilon_{g}>0\right)$ and semimetals $\left(\varepsilon_{g}<0\right)$. Below it is used to describe the crystals with and without the symmetry center.

These arguments suggested an idea to use the mentioned model for the comparative analysis of two materials within the framework of joint description. Let us define the main goal of such analysis as the investigation of the influence of symmetry center losses on the feature of the energy band structures and properties of carriers in the relevant energy bands.

\section{Basic equations and way of calculations}

The dispersion law has the following form within the model [3], if to apply the spherical system of coordinates $(k, \theta, \varphi)$ : 
$\left(\Gamma(\varepsilon)-(P k)^{2}\left(f_{1}(\varepsilon) \sin ^{2} \theta+f_{2}(\varepsilon) \cos ^{2} \theta\right)\right)^{2}-$ $-(P k)^{2} f_{3}^{2}(\varepsilon) \sin ^{2} \theta=0$.

The same expression has somewhat different form if to use the cylindrical system of coordinates $\left(k_{z}, k_{x y}, \varphi\right)$ :

$\left(\Gamma(\varepsilon)-P^{2}\left(f_{1}(\varepsilon) k_{x y}^{2}+f_{2}(\varepsilon) k_{z}^{2}\right)\right)^{2}-$

$-P^{2} f_{3}^{2}(\varepsilon) k_{x y}^{2}=0$.

Four polynomials for the energy $\varepsilon$ are used in the identical forms:

$$
\begin{aligned}
& \Gamma(\varepsilon)=\left[\left(\varepsilon-\varepsilon_{g}\right)\left(\left(\varepsilon+\frac{2 \Delta}{3}\right)\left(\varepsilon+\delta+\frac{\Delta}{3}\right)-\frac{2 \Delta^{2}}{9 \eta^{2}}\right)-\right. \\
& \left.-d^{2}\left(\varepsilon+\frac{2 \Delta}{3}\right)\right], \\
& f_{1}(\varepsilon)=\left(\varepsilon+\frac{\Delta}{3}\right)\left(\varepsilon+\delta+\frac{\Delta}{3}\right)-\frac{\Delta^{2}}{9 \eta^{2}}, \\
& f_{2}(\varepsilon)=\varepsilon\left(\varepsilon+\frac{2 \Delta}{3}\right) \eta^{-4}, \\
& f_{3}(\varepsilon)=\frac{2 \varepsilon \Delta d}{3 \eta} .
\end{aligned}
$$

There, $\varepsilon_{g}, \Delta, P$ are three well-known Kane's parameters [4] (the energy gap, spin-splitting parameter and matrix element of the pulse); $\delta$ is the known parameter of the crystal field [5] and $d$ is another parameter of the crystal field, which describes the absence of the symmetry center [3]; $\eta$ is the scalar factor taking into account the lattice deformation (mentioned and described above). The zero of energy ( $\varepsilon=0$ ) is united either with the top of the band of heavy holes (if $\varepsilon_{g}>0$ ) or with the bottom of the conduction band (if $\varepsilon_{g}<0$ ).

It is noticeable that Eqs (1), (2) are independent $\operatorname{on} \varphi$. Therefore, the surfaces of the equal energy are surfaces of rotation around the main crystal axis that is the polar axis, too [3].

The right parts of Eqs (1) and (2) have the trivial forms as product of two factors (let it be $P_{\alpha}(\varepsilon, k, \theta), P_{\beta}(\varepsilon, k, \theta)$ for Eq. (1), and $P_{\alpha}\left(\varepsilon, k_{z}, k_{x y}\right)$, $P_{\beta}\left(\varepsilon, k_{z}, k_{x y}\right)$ for Eq. (2)), because each of them is a difference of two quadratic items. For instance, Eq. (1) can be rewritten:

$$
P_{\alpha}(\varepsilon, k, \theta) P_{\beta}(\varepsilon, k, \theta)=0 \text {. }
$$

Here

$$
\begin{aligned}
& P_{\alpha}(\varepsilon, k, \theta)=\Gamma(\varepsilon)-(P k)^{2}\left(f_{1}(\varepsilon) \sin ^{2} \theta+\right. \\
& \left.+f_{2}(\varepsilon) \cos ^{2} \theta\right)-P k f_{3}(\varepsilon) \sin \theta, \\
& P_{\beta}(\varepsilon, k, \theta)=\Gamma(\varepsilon)-(P k)^{2}\left(f_{1}(\varepsilon) \sin ^{2} \theta+\right. \\
& \left.+f_{2}(\varepsilon) \cos ^{2} \theta\right)+P k f_{3}(\varepsilon) \sin \theta .
\end{aligned}
$$

The polynomials $P_{\alpha}\left(\varepsilon, k_{z}, k_{x y}\right), P_{\beta}\left(\varepsilon, k_{z}, k_{x y}\right)$ are structurally similar without any doubts.

Let us note, that $P_{\alpha}=P_{\beta}$ primarily if $f_{3}(\varepsilon)=0$. These conditions might be fulfilled for any energy only as for the crystal modifications with the symmetry center (because $d=0$ in this case). Therefore, each energy level should be twice degenerated. Obviously, it ought to be the well-known Kramers' degeneration. If $d \neq 0$, then $P_{\alpha} \neq P_{\beta}$ and these are the conditions for the phases without the symmetry center. Now the equations like $P_{\alpha}=0$ and $P_{\beta}=0$ have different solutions and the mentioned above degeneration is over.

Factorization (7) allows decreasing the orders of the dispersion equations. For instance, the equations $P_{\alpha}=0$ and $P_{\beta}=0$ have the fourth order for functions $\varepsilon_{\alpha, \beta}(k, \theta)$ (or $\varepsilon_{\alpha, \beta}\left(k_{z}, k_{x y}\right)$ ). These direct solutions might be obtained even in radicals, although proper expressions are quite cumbersome and unbelievably long. This circumstance seems to make no impression on a computer, in a contrast with its users. Someone can get indirect (implicit) solutions, too. This function $\varepsilon_{\alpha, \beta}(\varepsilon, \theta)$ or anything like to $\left(k_{x y}\left(\varepsilon, k_{z}\right), k_{x y}\left(\varepsilon, k_{x y}\right)\right)$ within the cylindrical system of coordinates, for which the equations (8), (9) are quadratic. These indirect solutions can be also useful as the direct solutions, being at the same time much shorter and simpler. What is more, they can serve to verification of any conclusions attained by using the direct solutions.

For instance, the velocity of carriers may be obtained simply as the gradient of a direct solution for their energies $\varepsilon_{\alpha, \beta}(k, \theta)$ :

$\mathbf{v}_{\alpha, \beta}=\frac{1}{\hbar} \operatorname{grad}_{\mathbf{k}}\left(\varepsilon_{\alpha, \beta}(k, \theta)\right)$.

On the other hand, the same result may be obtained from indirect solutions, or even from functions $P_{\alpha}(\varepsilon, k, \theta), \quad P_{\beta}(\varepsilon, k, \theta)$, using the well-known mathematical technique of the implicit derivatives.

Our calculations were leaning mostly on the presented above collection of simple expressions as well as on the following limited set of numerical parameters built-in into these (see Table 1).

It did not matter how bulky and lengthy may be some analytic expressions for a calculated result, on occasion. The main focus of the attention for us was to be in that point to represent such results in the forms of 
Table. Parameters of materials.

\begin{tabular}{|l|c|c|c|c|c|c|c|c|}
\hline Parameter & $\varepsilon_{g}, \mathrm{eV}$ & $\Delta, \mathrm{eV}$ & $P, \mathrm{eV} \cdot \mathrm{m}$ & $\delta, \mathrm{eV}$ & \multicolumn{2}{|c|}{$d, \mathrm{eV}$} & \multicolumn{2}{|c|}{$\eta$} \\
\hline Material & & & & & $D_{4 h}$ & $C_{4 v}$ & $D_{4 h}$ & $C_{4 v}$ \\
\hline $\mathrm{Zn}_{3} \mathrm{As}_{2}$ & 0.98 & 0.26 & $7.6 \cdot 10^{-10}$ & 0.07 & 0 & 0.035 & 1.00218 & 1.00352 \\
\hline $\mathrm{Cd}_{3} \mathrm{As}_{2}$ & -0.13 & 0.30 & $7.2 \cdot 10^{-10}$ & 0.09 & 0 & 0.035 & 1.00471 & 1.00565 \\
\hline
\end{tabular}

graphic dependences, evident and relatively simple. That is why this paper may be evaluated by someone as some illustrative overloading, although only on the face of it, how we are hoping.

\section{The results and discussions}

An old and good tradition requires the demonstration of the dependences of the carrier energy versus the module of the wave vector, or rarer of the pulse, under the condition of an unchanged direction. It is shown in Fig. 1 as the dependences $\varepsilon_{n}(k)$. It corresponds to the rectilinear motion of a carrier with some tangential acceleration, but in the absence of a normal acceleration. Both graphics present only the substantial part of the set, which consist of four energy bands, as a whole. So, the Fermi level is located anywhere either within or nearby presented pair of the energy bands. It is the pair of the bands of heavy and light holes, of course. Although it would be better to replace a term "heavy holes" by "heavy carries" as for cadmium arsenide.

There is the visible splitting of two sub-bands with the opposite spin states, which are located on both sides from a middle line (the sets of cross-like or circle-like points). These middle lines are the twice degenerated energy bands for the structures with the symmetry centers. This splitting is obviously caused by disappearing the symmetry center after the $4 / m m m\left(D_{4 h}\right) \rightarrow 4 m m\left(C_{4 v}\right)$ transition and, thus, it is a result of the removal of Kramers' degeneration.

The dependences of the magnitudes of this splitting on the module of the wave vector as well as its dependences on direction (the polar graphic) are shown for all bands in Fig. 2. In this figure, shown are the dependences for cadmium arsenide. However, the same dependences for zinc arsenide are exceedingly similar to those presented in Fig. 2, even in shallow details. The opposite sign of such splitting literally "strikes the eyes" as for one of these bands. Such a feature characterizes the bands of light holes of both materials. The maxima of the splitting magnitude correspond to $\theta=\pi / 2$ that describes the directions normal to the main crystal axis, whereas along this axis the splitting is absent. Even the greatest magnitudes of them are rather moderate and do not exceed a few hundred stakes of electron-volts, because the same order has the relevant parameter $d$ (see Table).

Other, and in a manner reverse approach look as if also reasonable from the physical point of view. That is study of solely cyclic motion of a carrier with some normal acceleration, but without any tangential
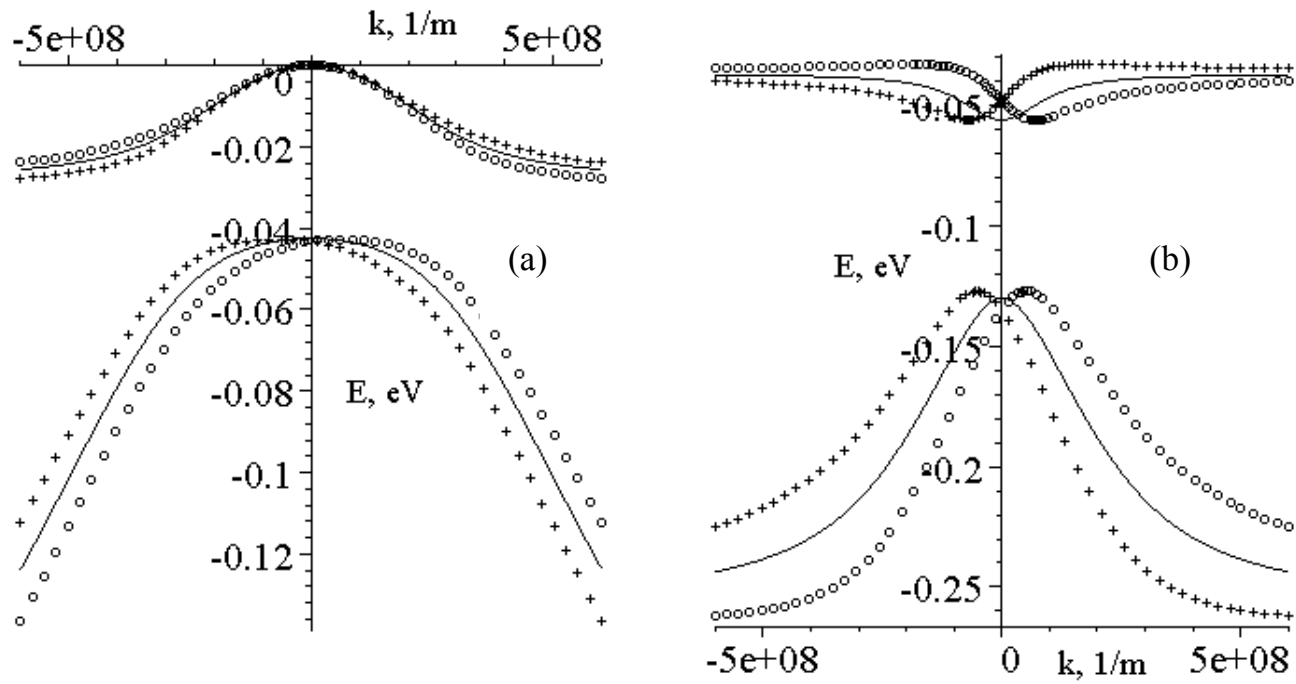

Fig. 1. The dependences $\varepsilon_{n}(k)$ along the $\theta=\pi / 2$ directions: for $\mathrm{Zn}_{3} \mathrm{As}_{2}$ (a) and $\mathrm{Cd}_{3} \mathrm{As}_{2}$ (b). 
(a)

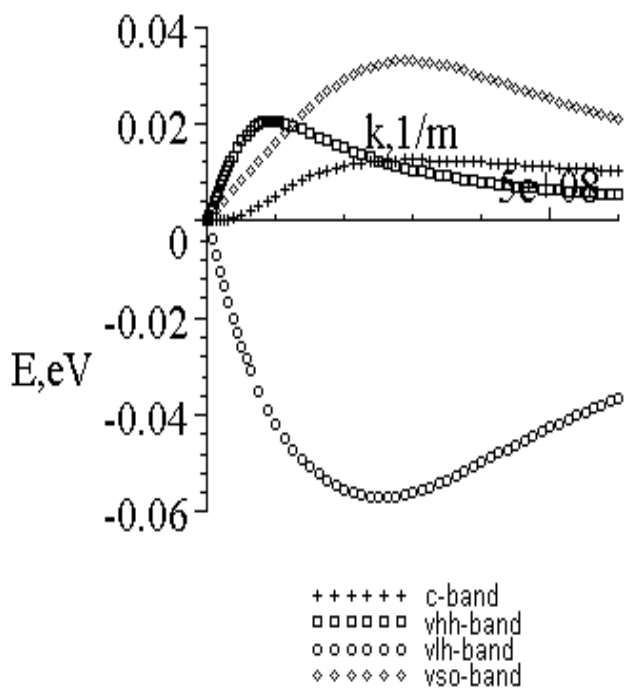

(b)

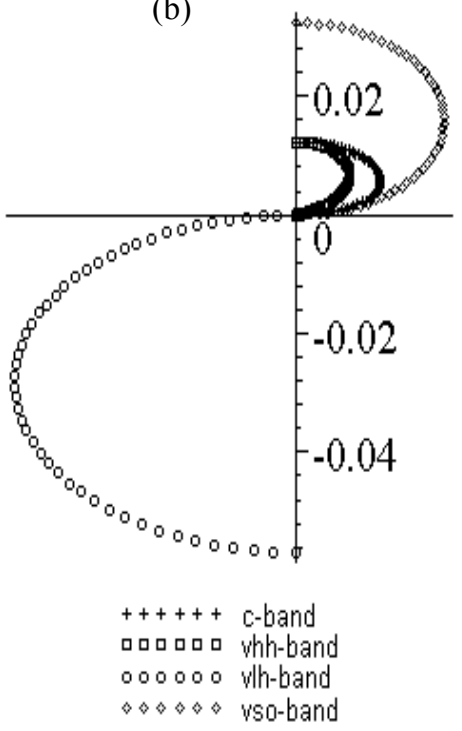

Fig. 2. The dependences of the spin splitting magnitude $v s$ the module of the wave vector (a) and $v s$ the direction (b), supposing that the horizontal axis of the polar plot (b) coincides with that for the main crystal axis.

components. At that rate, everyone would be interested in the dependences of the energy versus the direction of the carrier wave vector under the condition of its invariable module. These dependences are shown in Fig. 3 as curves $\varepsilon_{n}(\theta)$ for the same pair of bands as in Fig. 1. All symbols of Fig. 3 mean the same as those in Fig. 1. It is well seen from comparison of the dependences in Figs 1 and 3 that they seem to be very different in Fig. 1, while in Fig. 3 they are practically indistinguishable. Second observation is that energy anisotropy on the spherical surface $|k|=$ const is much greater for the heavy carriers.
If examine the modules of the carrier velocities, it ought to be keeping in mind, that the heavy and light holes are the major carriers only in zinc arsenide. Opposite, the light and heavy electrons are the same in cadmium arsenide. Let us begin with zinc arsenide.

The velocities of the both different kinds of the holes are almost comparable by the magnitudes along the directions, which is normal to main crystal axis. Nevertheless, the directions that are close to the direction of the main axis $(\theta \approx 0)$ are characterized by another and sharply different relations of these velocities. The anisotropy ought to be very strong as for
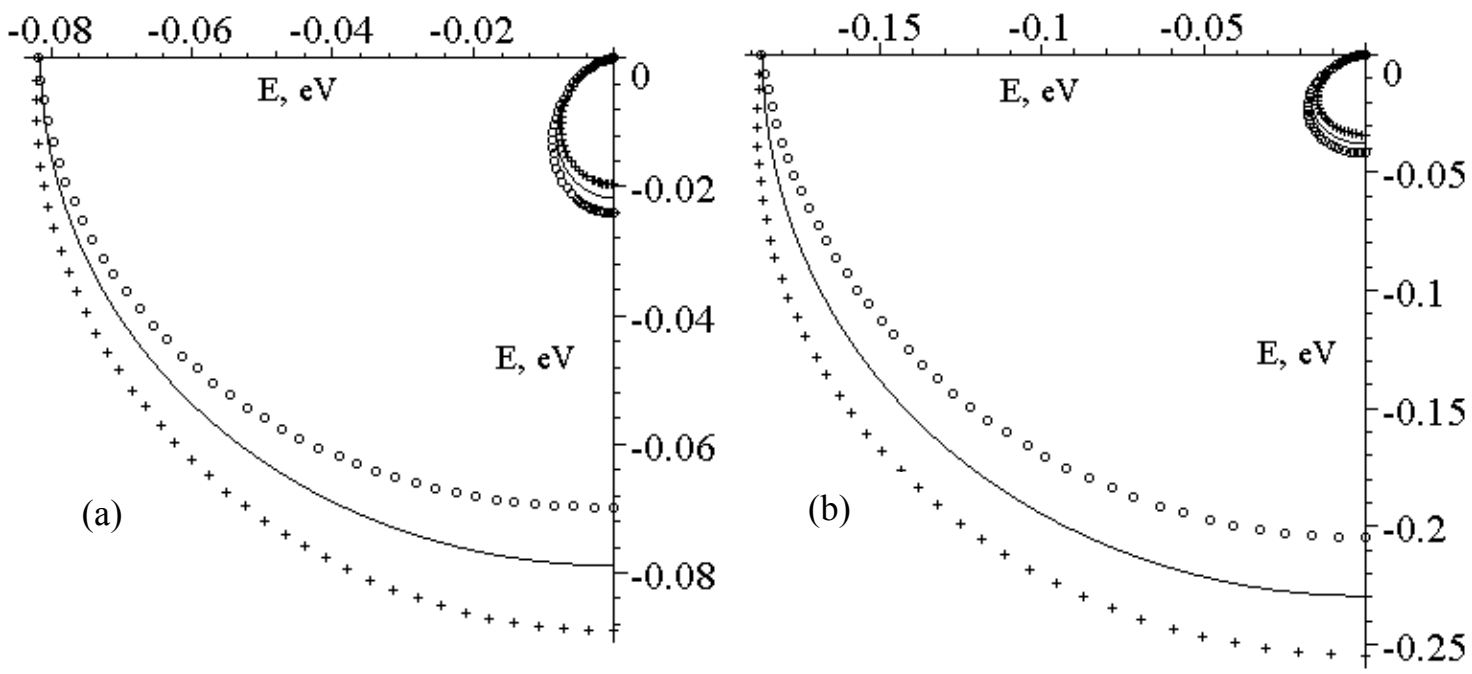

Fig. 3. The polar plot of the dependences $\varepsilon_{n}(\theta)$ for $\mathrm{Zn}_{3} \mathrm{As}_{2}$ (a) and $\mathrm{Cd}_{3} \mathrm{As}_{2}$ (b). 
the heavy holes, which may be extremely slow when moving along any direction close to the main axis. Furthermore, their velocities decrease with the energy in contrast to the light holes.

The spin splitting of the carrier velocities is maximal along the directions normal to the main crystal axis. In other words, the modules of velocities of holes with an identical spin are quite not identical at motion back and forth along almost any direction, in particular, in the plane normal to the main crystalline axis. This is the obvious and direct consequence of the symmetry center loss. Opposite, the holes with the opposite spins will be never assorted by their velocities when moving along this axis.

Below we consider the following material cadmium arsenide. The data is presented by Figs 5 and 6 separately. In particular, Fig. 5 illustrates the absolutely another behavior of the heavy carriers as compared to that shown in Fig. 4a for zinc arsenide. It may be explained taking into consideration first of them clear difference between the shapes of the equal energy surfaces (SEE) for heavy carriers.

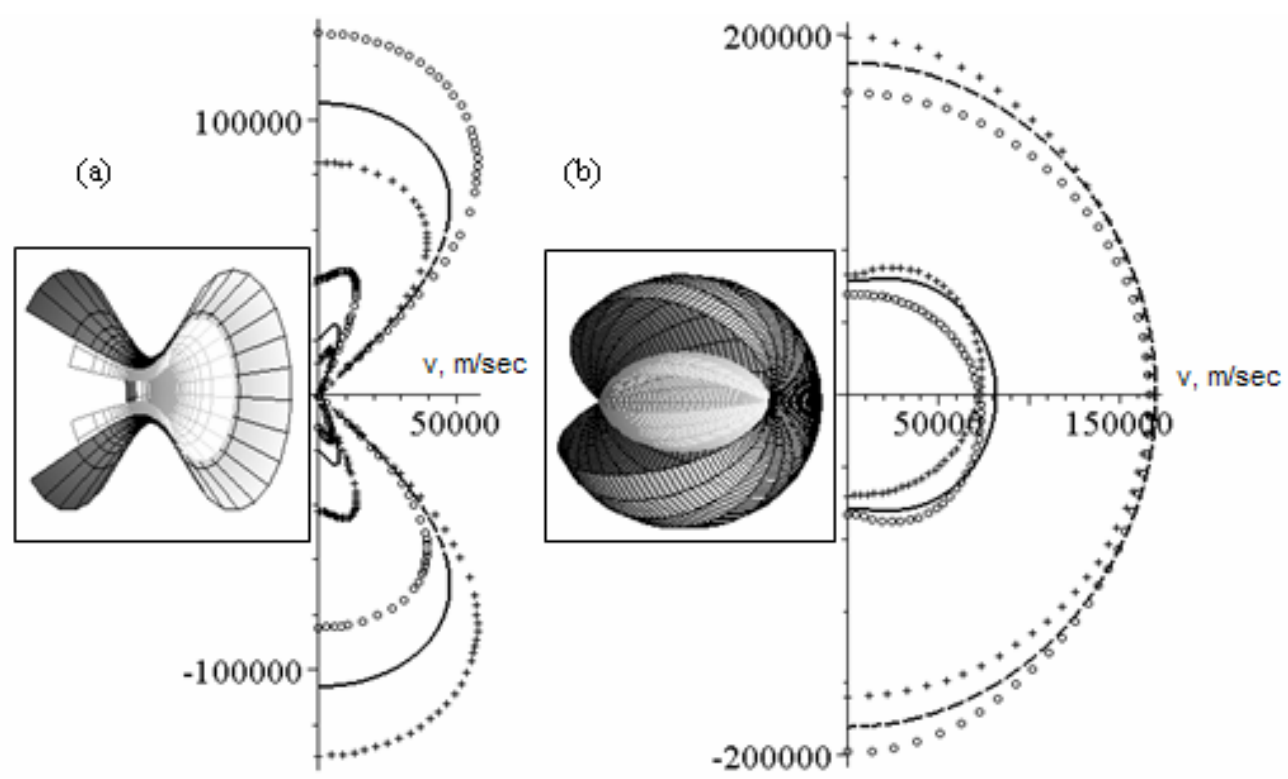

Fig. 4. Zinc arsenide: the polar plot of the velocity modules $v$ s the direction for heavy holes (a) at the energies -25 and $-15 \mathrm{meV}$, as well as for light holes (b) at -45 and $-55 \mathrm{meV}$. The insertions remind about typical geometry and topology of surfaces of the equal energy.

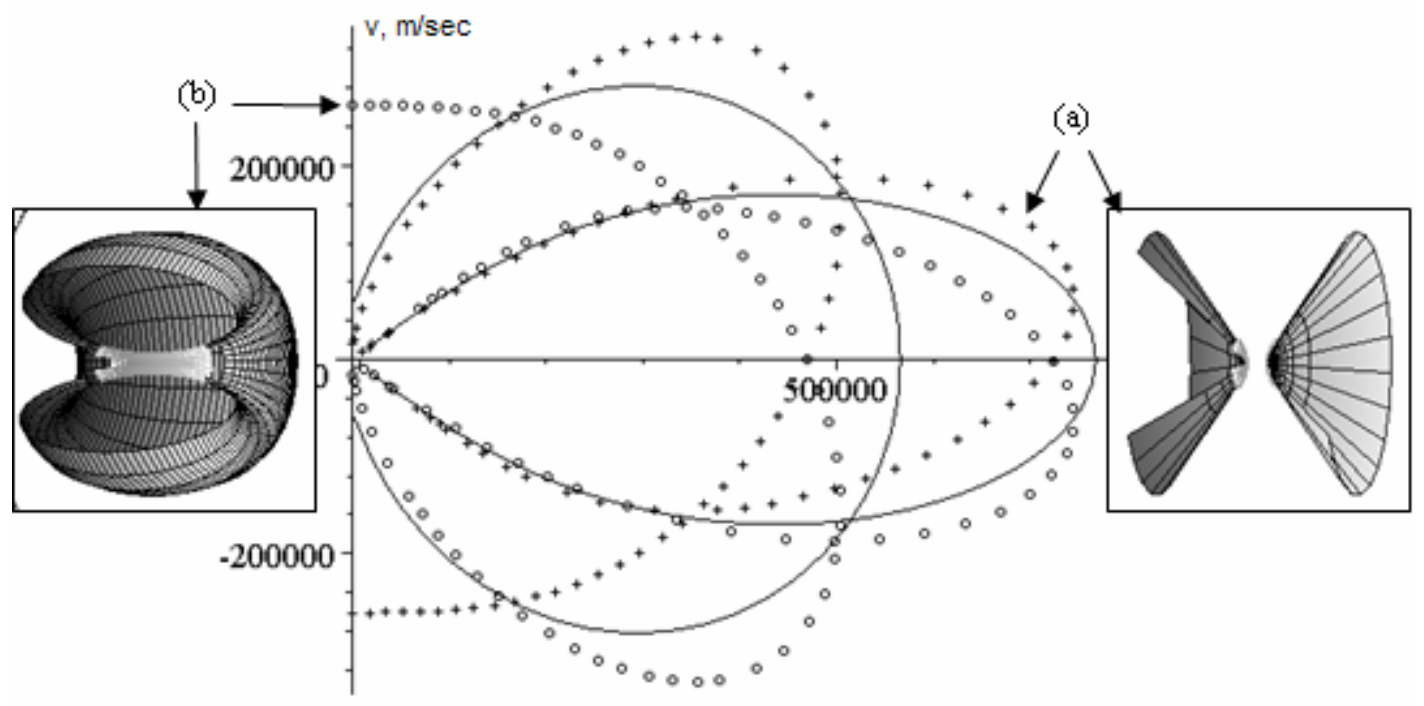

Fig. 5. Cadmium arsenide: the polar plot of the velocity modules $v s$ the direction for heavy carriers at the energies -15 (a) and $-40 \mathrm{meV}(\mathrm{b})$. The inserts have the same meaning as above. 


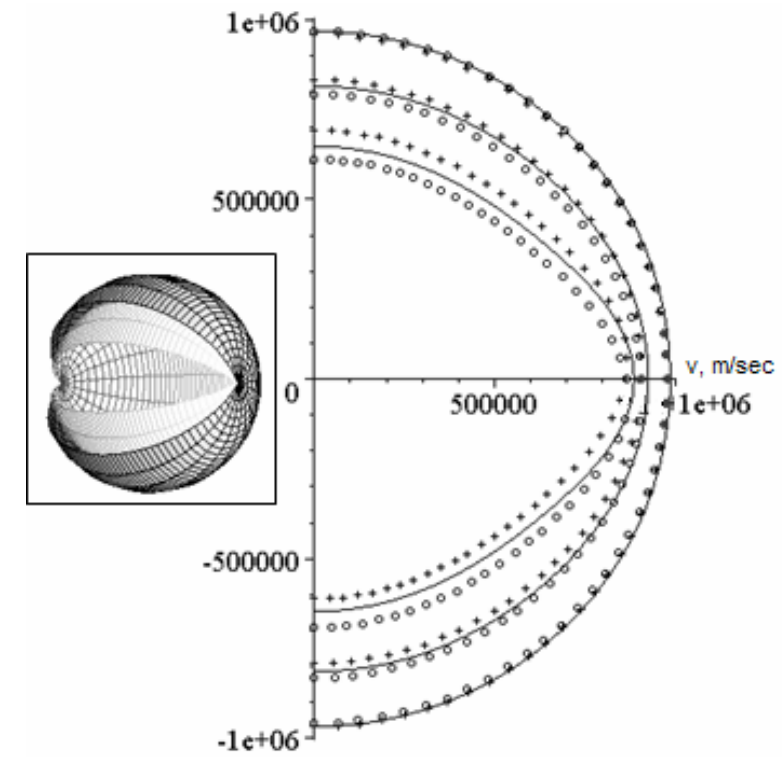

Fig. 6. Cadmium arsenide: the polar plot of the velocity modules $v s$ the direction for light electrons at the energies 25 , 50 and $120 \mathrm{meV}$, respectively.

Nevertheless, all these effects caused by the symmetry center loss are also present in this case, of course. Essential changing the topology and geometry of SEE, which occurs between -15 and $-40 \mathrm{meV}$ cannot abolish these general peculiarities, in spite of bringing some individuality into them. Both parts of the previous sentence are well visible in Fig. 5. Both the strong anisotropy of velocities and the possibility of separation of carriers by their spins are evidently also common features for heavy carriers of both materials.

The light electrons from the conduction band of cadmium arsenide demonstrate the dependences, which are very similar to analogous curves for light holes of zinc arsenide, in contrast to the heavy carriers. It becomes obvious from the comparison of Figs 4 and 6 . Let us note that the influence of the symmetry centre loss weakens with increasing the energy module so that such influence becomes quite insignificant as for the strong degenerated samples of both materials. In other words, the effects of absence of the symmetry centre need to be searched in the samples with the lower concentration of carriers and with the Fermi energies that are closer to the band extremes. The degenerated samples are insensitive to it.

\section{Conclusions}

The main results of our analysis are as follows:

1. The loss of the symmetry centre results in the splitting of the energy bands that are maximal along some direction normal to the main crystalline axis as well as for specific values of the wave vectors as for the different energy bands.

2. The velocities of the carriers with the same spins are clearly various in relation to the motion "back and forth" almost along every direction. Such features allow to separate carriers in accord with their spins, in principle.

3. The samples with the low concentration of carriers demonstrate the results of the absence of the symmetry centre better. Moreover, the velocities of heavy carriers are more sensitive in comparison with those of the light carriers, as well as the main carriers of $p-\mathrm{Zn}_{3} \mathrm{As}_{2}$ are more sensitive as compared with the electrons of $n-\mathrm{Cd}_{3} \mathrm{As}_{2}$ in the case of the absent symmetry centre.

4. The heavy carriers of both materials demonstrate more pronounced anisotropy of characteristics for light carriers.

\section{Acknowledgements}

Authors wish to thank Dr. Stanislaw Shutov for his helpful participation in the processes of preparation of this paper.

\section{References}

1. E.K. Arushanov, Crystal growth \& characterization of II-V compounds // Progr. Cryst. Growth \& Charact. 3, p. 211-255 (1980).

2. G.P. Chuiko, N.L. Don, V.V. Ivchenko, Ordering and polytypism in $\mathrm{A}_{3}^{\mathrm{II}} \mathrm{B}_{2}^{\mathrm{V}}$ crystals // Functional materials 12(3), p. 454-460 (2005).

3. G. Chuiko, N. Don, O. Dvornik, V. Ivchenko, A. Sergeev, Simple inverted band structure model for cadmium arsenide $\left(\mathrm{Cd}_{3} \mathrm{As}_{2}\right)$ // Moldavian Journ. of Phys. Sciences 2(1), p.88-94 (2003).

4. E.O. Kane, Band structure of indium antimonide // J. Phys. Chem. Solids 1, p. 249-261 (1957).

5. H. Kildal, Band structure of $\mathrm{Cd} \mathrm{GeAs} 2$ near $k=0 / /$ Phys. Rev. 10(12), p. 5082-5087 (1974). 